\title{
Nurses' Knowledge and Perceptions of Advance Care Planning, an Ethical Responsibility
}

\section{Savitri Singh-Carlson}

School of Nursing, Global Studies Institute, California State University, USA

*Corresponding author: Savitri Singh-Carlson, School of Nursing, Global Studies Institute, California State University, USA, Tel: +5622342432; E-mail: savitri.singhcarlson@csulb.edu

Received Date: August 15, 2017; Accepted Date: August 28, 2017; Published Date: September 08, 2017

Copyright: (C) 2017 Singh-Carlson S. This is an open-access article distributed under the terms of the Creative Commons Attribution License, which permits unrestricted use, distribution, and reproduction in any medium, provided the original author and source are credited.

\section{Introduction}

Discussions around advance care planning (ACP) do not mean making decisions about what to do at the end of life, but how to cope with disease and treatment. Studies suggest that HPs and patients are ambivalent when talking about death and end of treatments and often avoid these conversations. Results show that discussions around ACP with advanced cancer patients reduced patients' overly optimistic chances of survival, thereby reducing aggressiveness of medical care near death. Discussions around ACP offer patients opportunity to define their goals and expectations for the medical care they want to receive. Advance directives and living wills are tools of ACP that are written instructions regarding one's medical care preferences. Having open communication on patients' wishes on ACP is beneficial for patients. This informs them of medication and treatment plans that are available for them at the beginning of diagnosis, thereby allowing them to make informed decisions that lead to a better quality of life. In light of this evidence, exploring nurses' perceptions that are ingrained by their own beliefs and values around the use of advance care plans for planning end-of-life will help them examine their own ethical responsibility in their role as healthcare providers. This knowledge can be used for educating nurses who are future primary care providers. It is a professional responsibility, especially since many nurses with a graduate degree may work in oncology settings $[1,2]$.

\section{Description of Research}

\section{Purpose}

To explore nurses' knowledge and perceptions of advance care planning in relation to palliative care service. This study will help to facilitate practicing oncology nurses, current graduate nursing students and recently graduated advance practice nurses' understanding of their ethical responsibility to inform and educate patients and families on the benefits of advance care planning.

\section{Research questions}

What are nurses' professional attitudes and beliefs on the use of advanced care planning in their clinical setting?

Are there any differences in professional attitudes and beliefs amongst registered nurses and specialized oncology nurses on the use of advanced care planning in their clinical setting?

\section{For Canadian participants (participants must meet all criteria to qualify)}

- $22-70$ years of age
- Male or female

- Be a member of the Canadian Association of Nurses in Oncology (CANO)

- A Registered Nurse

- Currently working in an oncology setting and in a role of direct patient cancer care

- Research design and methodology

Convenience sample of oncology nurses, current graduate nursing students, and recently graduated advanced practice nurses will be recruited from the Oncology Nursing Society, Canadian Association of Nursing Oncology, and School of Nursing at CSULB. After IRB approval, invitations to be part of the study will be emailed to all potential participants using a web-based survey called Fluid survey $[3,4]$.

This study will employ a mixed methods approach with responses to choose from with some written responses, which is a fit into providing an insight into nurses' understanding of their ethical responsibility in discussions surrounding ACP. The survey from Advance Care Planning, Australia (http://advancecareplanning.org.au/) is adapted to fit the Canadian and U.S healthcare system, with the content of the questions remaining the same. Permission has been granted to use this Advance Care Planning Service survey with commitment to acknowledge Advance Care Planning, Australia. The survey will determine nurses' professional attitudes and beliefs on their use of advance care planning with patients in the clinical setting. Content analysis will be used to analyze the qualitative content in order to provide an in-depth understanding of participants' knowledge and perceptions of ACP and their personal meaning attached to this topic in light of their professional and personal social/cultural influences. SPSS will be used to analyze the inferential and descriptive data from the Fluid surveys. Currently there are between 200-300 graduate/ alumni nursing students in a part/full time status. It is hoped that a total of approximately 600 participants will respond to the survey. A letter of reminder will be sent after 4 weeks.

\section{Reference}

1. Johnstone MJ and Kanitsaki O (2009) Ethics and advance care planning in a culturally diverse society. Journal of transcultural nursing 20: 405-416.

2. Puntillo KA and McAdam JL (2006) Communication between physicians and nurses as a target for improving end-of-life care in the intensive care unit: challenges and opportunities for moving forward. Critical care medicine 34: S332-S340.

3. Ulrich CM, Taylor C, Soeken K, O’Donnell P, Farrar A, et al. (2010) Everyday ethics: ethical issues and stress in nursing practice. Journal of advanced nursing 66: 2510-2519. 
Citation: Carlson SS (2017) Nurses' Knowledge and Perceptions of Advance Care Planning, an Ethical Responsibility. J Integr Oncol 6: 197. doi:10.4172/2329-6771.1000197

Page 2 of 2

4. Maier-Lorentz MM (2008) Transcultural nursing: Its importance in nursing practice. Journal of cultural diversity 15: 37. 